\title{
AN ELASTO-VISCOPLASTIC MODEL FOR HEXAGONAL CLOSED PACKED MATERIALS: FORMULATION AND NUMERICAL IMPLEMENTATION
}

\author{
S.H.Wu ${ }^{1}, \dagger$, F.M. Andrade Pires ${ }^{1}$, Abel D. Santos ${ }^{1}$, A. Barata da Rocha ${ }^{1}$ \\ ${ }^{1}$ Faculty of Engineering, University of Porto
}

Rua Dr. Roberto Frias 4200-465 Porto, Portugal

†e-mail: shwoo2001@gmail.com

\begin{abstract}
Titanium alloys have widespread applications in defense and aerospace industries owing to its high performance mechanical properties, particularly its high strength to weight ratio [1, 2, and 5]. Due to their Hexagonal closed packed (HCP) crystallographic structure; they have anisotropic properties and high strength differential (SD) effects in tension and compression during deformation [3, 4]. In practical applications, titanium alloys might be subject to complex loading conditions, which may include the presence of various strain rates. Therefore, in order to study their visco-plastic mechanical behavior, the present contribution proposes an elastic-viscoplastic model for HCP materials by extending the Cazacu yield criteria with the widely used visco-plastic formulation proposed by Perzyna [9]. The new model is implemented in an implicit quasi-static finite element framework with a fully implicit integration scheme. The stress integration algorithm employs the closest point projection method (CPPM) combined with the line search method. Two benchmark tests are performed to study the material mechanical behavior of the Ti-6Al-4V alloy at various strain rates. The stability and accuracy of this model is analyzed.
\end{abstract}

Keywords: HCP materials, Cazacu model, constitutive modeling, Perzyna model

\section{INTRODUCTION}

Titanium alloys are extensively used in medical devices, defense applications, aerospace and automotive industries, because of their high comprehensive performance, superior specific strength to weight ratio, exceptional corrosion resistance, and biocompatibility etc. Titanium alloys have the microstructural arrangement of Hexagonal Closed Packed (HCP) crystallographic materials. Therefore, these materials will exhibit different mechanical behaviors when compared to other metals with FCC and BCC structures, such as steel, aluminum etc. At room temperature, there is a limited number of basal and non-basal slip systems to be activated to accommodate general deformation, so the activation of twinning plays an important role to accommodate the deformation. The polar nature of deformation twinning pro- 
motes a strong asymmetry between yielding in tension and compression, usually known as strength differential effect (SD) [5, 7]. In addition, Jiang et al. [8] have shown that the volume fraction of contraction and double twins increases with strain rate, which indicates that twinning is an athermal deformation mode and has a high strain rate sensitive. Therefore, the dynamic behavior of Titanium alloys, particularly at high strain rates, must be modeled in order to design new applications. Although numerous yield criteria such as Hill, Hosford, Barlat, or Barlat and Lian, Karafillis et al $[1,4]$ have been proposed, all of them fail to capture this unconventional mechanical behavior. In order to model the response of HCP metals, Cazacu et al. [1, 3] studied the mechanical behavior of several HCP metals under monotonic loading paths and proposed two generic yield criteria. These yield functions employ the transformed stresses which are obtained from a 4th-order linear transformation operator on the Cauchy stress tensor. These two criteria were implemented in Cut Plane method by Nixon et al. [1] and Plunkett et al. [9], respectively.

In this contribution, an elasto-viscoplastic model for Hexagonal Closed Packed materials is formulated. The Perzyna overstress viscoplastic model is coupled with Cazacu yield criteria here and the resulting constitutive model is implemented within an implicit quasistatic finite element environment. In the numerical stress integration, a primal Closest Point Projection Method (CPPM) integration algorithm and a closed form consistent tangent operator are derived. Iso-error maps are used to illustrate the accuracy and stability of the algorithm at different strain rates.

\section{FORMULATION OF ELASTO-VISCO PLASTIC MODEL}

In the infinitesimal strain domain, similar to the classical plastic model, the total strain rate, $\dot{\varepsilon}$, in an elasto-viscoplastic material point can be additively decomposed into an elastic component, $\dot{\varepsilon}^{e}$, and a viscoplastic component, $\dot{\varepsilon}^{v p}$ :

$$
\dot{\varepsilon}=\dot{\varepsilon}^{e}+\dot{\varepsilon}^{v p},
$$

where the superimposed dot denotes the time derivative. The stress rate, $\dot{\boldsymbol{\sigma}}$, is related to the strain rate via the constitutive relation

$$
\dot{\sigma}=\mathrm{D}^{\mathrm{e}}: \dot{\varepsilon}^{e}
$$

with $\mathbf{D}^{\mathbf{e}}$ the fourth-order tensor containing the tangential elastic stiffness moduli. Furthermore, the viscoplastic strain rate evolves via a flow rule,

$$
\dot{\boldsymbol{\varepsilon}}^{v p}=\dot{\gamma} N \text {. }
$$

The scalar, $\dot{\gamma}$, is a non-negative parameter, known as the consistency parameter (Simo and Hughes, 1988).

In the past decades, numerous viscoplastic models $[10,11]$ have been proposed. Perzyna's overstress theory provides a unified approach to analyze a wide range of engineering problem and has been widely used to capture rate-dependent effects in solid materials, like Luders bands and the Portevin-Le Chatelier effect in metals, shear banding and creep in geomaterials and to analyze localization and bifurcation properties. Owing to the strain rate sensitivity of the twinning deformation mechanism for HCP materials, there is a dependence 
of mechanical behavior with the strain rate.

In Perzyna's model, the consistency parameter is expressed by

$$
\dot{\gamma}=\frac{\langle\Phi(F)\rangle}{\eta}
$$

where $\eta$ is the viscosity parameter, $\Phi$ represents the overstress function that depends on the rate-independent yield surface $F$ and " $\langle\cdot\rangle$ " are the McCauley brackets, such that:

$$
\langle\Phi(F)\rangle= \begin{cases}\Phi(F), & \text { if } \Phi(F) \geq 0 \\ 0, & \text { if } \Phi(F)<0\end{cases}
$$

\subsection{Yield criteria}

The yield function proposed by Cazacu et al.[1] was obtained by extending the orthotropic Drucker's isotropic yield criterion with a constructed generalizations of the invariants of the deviatoric stress. The original Cauchy stress $\{\boldsymbol{\sigma}\}$ is transformed to a transformed tensor $\{\boldsymbol{\Sigma}\}$, which is defined as

$$
\Sigma_{i j}=L_{i j k l} \sigma_{k l}
$$

The fourth-order linear transformation operator $(\mathbf{L})$ can be represented in $6 \times 6$ matrix format as

$$
\mathbf{L}=\left[\begin{array}{cccccc}
\frac{a_{2}+a_{3}}{3} & -\frac{a_{3}}{3} & -\frac{a_{2}}{3} & 0 & 0 & 0 \\
-\frac{a_{3}}{3} & \frac{a_{1}+a_{3}}{3} & -\frac{a_{1}}{3} & 0 & 0 & 0 \\
-\frac{a_{2}}{3} & -\frac{a_{1}}{3} & \frac{a_{1}+a_{2}}{3} & 0 & 0 & 0 \\
0 & 0 & 0 & a_{4} & 0 & 0 \\
0 & 0 & 0 & 0 & a_{5} & 0 \\
0 & 0 & 0 & 0 & 0 & a_{6}
\end{array}\right]
$$

Cazacu's anisotropic yield function can be expressed by

$$
F=A\left(J_{2}^{0 \frac{3}{2}}-c J_{3}^{0}\right)^{1 / 3}-\sigma_{y},
$$

where, $J_{2}^{0}$, is the second invariant of the deviatoric transformed stress, and $J_{3}^{0}$, is the third invariant of deviatoric transformed stress, respectively, which can be obtained as

$$
\begin{aligned}
& J_{2}^{0}=\frac{1}{2} \operatorname{tr}\left(\Sigma^{2}\right), \\
& J_{3}^{0}=\frac{1}{3} \operatorname{tr}\left(\Sigma^{3}\right) .
\end{aligned}
$$

The constant $A$ is defined as

$$
A=3\left[\left(a_{2}^{2}+a_{3}^{2}+a_{2} a_{3}\right)^{\frac{3}{2}}-c\left(a_{2}+a_{3}\right) a_{2} a_{3}\right]^{-1 / 3},
$$

where $c$ is a material parameter, which can be expressed in terms of the yield in uniaxial tension, $\sigma_{T}$, and the yield in uniaxial compression, $\sigma_{c}$, as 


$$
c=\frac{3 \sqrt{3}\left(\sigma_{T}^{3}-\sigma_{c}^{3}\right)}{2\left(\sigma_{T}^{3}-\sigma_{c}^{3}\right)}
$$

To ensure the convexity of the yield function, the material constant $c$ is limited to the range $\left[-\frac{3 \sqrt{3}}{2}, \frac{3 \sqrt{3}}{4}\right]$.

\section{STRESS INTEGRATION}

In this section, the evolution of the different quantities from time $t_{n}$ to time $t_{n+1}=t_{n}+\Delta t$ at each integration point will be treated with Closet Point Projection Method (CPPM) stress integration algorithm. Within a typical time interval $\left[t_{n}, t_{n+1}\right]$, it is assumed that all variables $\left\{\boldsymbol{\sigma}_{n}, \boldsymbol{\varepsilon}_{n}^{e}, \boldsymbol{\varepsilon}_{n}^{p}, \bar{\varepsilon}_{n}^{p}\right\}$ at time $t_{n}$ are known, and the stress together with the updated variables, which characterize the inelastic response of the material, are pursued for given strain increment $\Delta \boldsymbol{\varepsilon}$. The numerical integration of elasto-plastic constitutive equations is typically carried out by means of the so-called elastic predictor return mapping schemes. A fully implicit elastic predictor return mapping method, which is called Closest Point Projection Method (CPPM), is implemented within an implicit quasi-static finite element environment.

In order to integrate the governing equations in a similar means to generalized plasticity, the overstress Perzyna model is chosen. The strain rate is assumed to be computed by $\dot{\bar{\varepsilon}}^{p}=$ $\Delta \bar{\varepsilon} / \Delta t$

The summary of the CPPM for Visco-Cazacu model is presented in Box.1. In order to obtain quadratic convergence in the solution of an initial boundary value problem within an implicit finite element environment, the tangent operator consistent with the general algorithm is needed to assemble the tangent stiffness matrix. The fourth-order tensor can be obtained by computing the derivative of the updated stress tensor $\boldsymbol{\sigma}_{n+1}$ with respect to the final strain $\boldsymbol{\varepsilon}_{n+1}$. When the stress state lies within the elastic domain and no viscoplastic flow occurs, the tangent operator is the elastic tangent operator $\mathbf{D}^{\mathbf{e}}$. Otherwise, the elasto-viscoplastic consistent tangent operator has to be derived by consistently linearising the viscoplastic return-mapping algorithm under viscoplastic flow. The closed form for the viscoplastic tangent operator can be expressed by

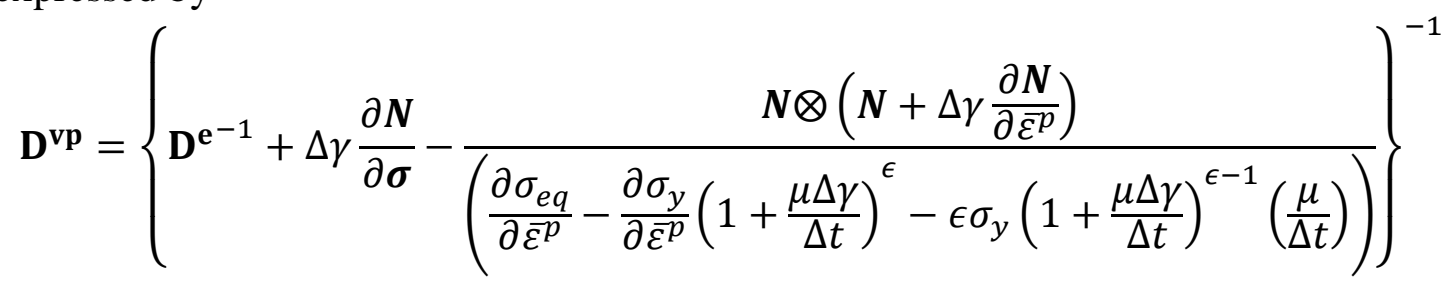

It is important to remark that this tangent operator is not symmetric. 


\section{Box 1. Fully implicit closest point projection method (CPPM)}

(i) Elastic predictor. Given $\Delta \boldsymbol{\varepsilon}$ and the state variables at $t_{n}$, evaluate the elastic trial state:

$$
\begin{aligned}
& \boldsymbol{\varepsilon}_{n+1}^{e \text { trial }}=\boldsymbol{\varepsilon}_{n}^{e}+\Delta \boldsymbol{\varepsilon} ; \bar{\varepsilon}_{n+1}^{p \text { trial }}=\bar{\varepsilon}_{n}^{p} \\
& P_{n+1}^{\text {trial }}=K \varepsilon_{v n+1}^{\text {etrial }} ; \boldsymbol{S}_{n+1}^{\text {trial }}=2 G \boldsymbol{\varepsilon}_{d n+1}^{\text {etrial }} ; \boldsymbol{\sigma}_{n+1}^{\text {trial }}=\boldsymbol{S}_{n+1}^{\text {trial }}+P_{n+1}^{\text {trial }} \boldsymbol{I} \\
& \Sigma_{n+1}^{\text {trial }}=\mathbf{L} \boldsymbol{\sigma}_{n+1}^{\text {trial }} \\
& J_{2 n+1}^{0 \text { trial }}=\frac{1}{2} \operatorname{tr}\left(\Sigma_{n+1}^{\text {trial }^{2}}\right) ; J_{3 n+1}^{0 \text { trial }}=\frac{1}{3} \operatorname{tr}\left(\Sigma_{n+1}^{\text {trial }^{3}}\right) ; \\
& \sigma_{\text {eq } n+1}^{\text {trial }}=A_{1}\left[\left(J_{2 n+1}^{0 \text { trial }}\right)^{\frac{3}{2}}-c J_{3 n+1}^{0 \text { trial }}\right]^{1 / 3}
\end{aligned}
$$

(ii) Check Plastic admissibility

$$
\begin{aligned}
& \text { If } F=\sigma_{e q n+1}^{\text {trial }}-\sigma_{y}\left(\bar{\varepsilon}_{n+1}^{\text {trial }}\right) \leq 0 \\
& \text { Then set }()_{\mathrm{n}+1}=()_{\mathrm{n}+1}^{\text {trial }} \text { and EXIT }
\end{aligned}
$$

(iii)Return mapping. Solve the system of eight equations using the Newton-Raphson iterative method

$$
\begin{gathered}
\left\{\begin{array}{c}
\boldsymbol{\varepsilon}_{n+1}^{e}-\boldsymbol{\varepsilon}_{n+1}^{e \text { trial }}+\Delta t \boldsymbol{G}\left(\boldsymbol{\sigma}_{n+1}, \bar{\varepsilon}_{n+1}^{p}\right) \\
\bar{\varepsilon}_{n+1}^{p}-\bar{\varepsilon}_{n}^{p}+\Delta t J\left(\boldsymbol{\sigma}_{n+1}, \bar{\varepsilon}_{n+1}^{p}\right) \\
\sigma_{e q n+1}-\sigma_{y n+1}\left(1+\frac{\mu \Delta \gamma_{n+1}}{\Delta t}\right)
\end{array}\right\}=\left\{\begin{array}{l}
\mathbf{0} \\
0 \\
0
\end{array}\right\} \\
\boldsymbol{G}\left(\boldsymbol{\sigma}_{n+1}, \bar{\varepsilon}_{n+1}^{p}\right)=\dot{\gamma} \boldsymbol{N}_{n+1}\left(\boldsymbol{\sigma}_{n+1}, \bar{\varepsilon}_{n+1}^{p}\right), \quad J\left(\boldsymbol{\sigma}_{n+1}, \bar{\varepsilon}_{n+1}^{p}\right)=\dot{\gamma} H\left(\boldsymbol{\sigma}_{n+1}, \bar{\varepsilon}_{n+1}^{p}\right)=-\dot{\gamma} \\
\dot{\gamma}=\frac{1}{\mu}\langle\Phi(f)\rangle=\frac{1}{\mu}\left\langle\left(\frac{\boldsymbol{\sigma}_{n+1}, \bar{\varepsilon}_{n+1}^{p}}{\sigma_{y}\left(\bar{\varepsilon}_{n+1}^{p}\right)}\right)^{1 / \epsilon}-1\right\rangle \\
\boldsymbol{N}_{n+1}=\frac{\partial F}{\partial \boldsymbol{\sigma}}
\end{gathered}
$$

The solution is found for $\boldsymbol{\varepsilon}_{n+1}^{e}, \bar{\varepsilon}_{n+1}^{p}$ and $\Delta \gamma$. The stress tensor can be obtained by

$$
\boldsymbol{\sigma}_{n+1}=\mathbf{D}^{e}: \boldsymbol{\varepsilon}_{n+1}^{e}
$$

\section{(iv)EXIT}

\section{NUMERICAL EXAMPLE}

In this section, some numerical examples are presented to verify the implementation of the proposed elasto-viscoplastic constitutive model within an implicit quasi-static finite element environment. The numerical examples are divided into 2 sections with different objectives. In the first set of examples, the uniaxial tensile and uniaxial-compressive tests are performed to evaluate the asymmetric mechanical behavior at different strain rates. In the second set of 
examples, several iso-error maps are presented to investigate the accuracy of the CPPM stress integration scheme at different strain rates. The material constants, which are employed in all examples, are listed in Table 1.

Table.1 Material properties for Titanium [5]

\begin{tabular}{|c|c|c|c|}
\hline & $\rho\left(\mathrm{g} / \mathrm{cm}^{3}\right)$ & E,Modulus $(\mathrm{GPa})$ & $v$, Poisson's Ratio \\
\hline Titanium & 4.51 & 120000 & 0.361 \\
\hline
\end{tabular}

The anisotropy coefficient values of pure titanium corresponding to the yield surface evolution can be seen in Table 2 .

Table 2 - Anisotropy coefficient values of pure titanium corresponding to the yield surface evolution [5]

\begin{tabular}{|c|c|c|c|c|c|c|c|c|}
\hline Strain $\left(\bar{\varepsilon}^{p}\right)$ & $\begin{array}{l}\text { Yield strength } \\
(\mathrm{MPa})\end{array}$ & $a_{1}$ & $a_{2}$ & $a_{3}$ & $a_{4}$ & $a_{5}$ & $a_{6}$ & $c$ \\
\hline 0.0 & 208 & 0.5454 & 0.501 & 1.09 & 0.7246 & -0.8675 & -0.8675 & -0.2168 \\
\hline 0.025 & 245 & 0.5231 & 0.4745 & 0.9034 & 0.7309 & 0.7202 & 0.7202 & -0.2198 \\
\hline 0.05 & 261 & 0.6694 & 0.5585 & 1.103 & 0.9138 & 0.9381 & 0.9381 & -0.2291 \\
\hline 0.075 & 273 & 0.6960 & 0.5969 & 1.127 & 0.9838 & 0.9716 & 0.9716 & -0.2607 \\
\hline 0.1 & 284 & 0.5356 & 0.4768 & 0.8603 & 0.7761 & 0.7714 & 0.7714 & -0.2754 \\
\hline 0.2 & 317 & 0.061 & 0.0576 & 0.0869 & 0.087 & 0.0794 & 0.0794 & -0.5908 \\
\hline 0.4 & 270 & 0.0632 & 0.062 & 0.0788 & 0.0816 & 0.0801 & 0.0801 & -1.0330 \\
\hline 0.5 & 389 & 0.9547 & 0.957 & 1.2140 & 1.181 & 1.176 & 1.176 & -1.1480 \\
\hline
\end{tabular}

\subsection{Single element tests}

(a) Uniaxial tensile test

In this example, a uniaxial stress state is applied to a single 8-node brick element (with one integration point). The length of the element is $10 \times 10 \times 10 \mathrm{~mm}^{3}$. This example is conducted to demonstrate the ability of the finite element formulation to capture strain rate sensitivity. The element is stretched with a prescribed constant (in time) velocity, $v$, along the rolling direction (x direction). The geometry and the boundary conditions applied to the single element for the uniaxial tensile test in $\mathrm{x}$ direction are shown in Figure 1.

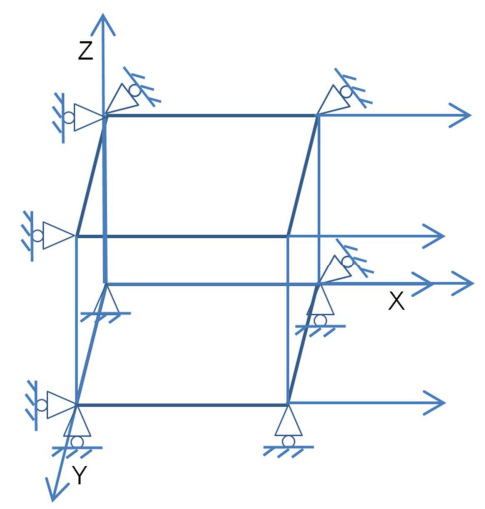

Figure.1 Uniaxial tensile test 
The normalized stretching rate can be defined conveniently for the unit cell by $v^{*}=\mu v$ and the simulation is carried out for three different values of $v^{*}$

$$
v^{*}=10^{-4}, 10^{0}, 10^{4} \text {. }
$$

This choice covers very slow to very fast strain rates and is meant to demonstrate the robustness of the integration algorithm over a wide range of strain rates. In order to show the effect of the rate-sensitivity parameter on the behavior of the model, in the uniaxial-tensile stress state, four values of $\epsilon$ are considered

$\epsilon=10^{1}, 10^{0}, 10^{-2}, 10^{-6}$

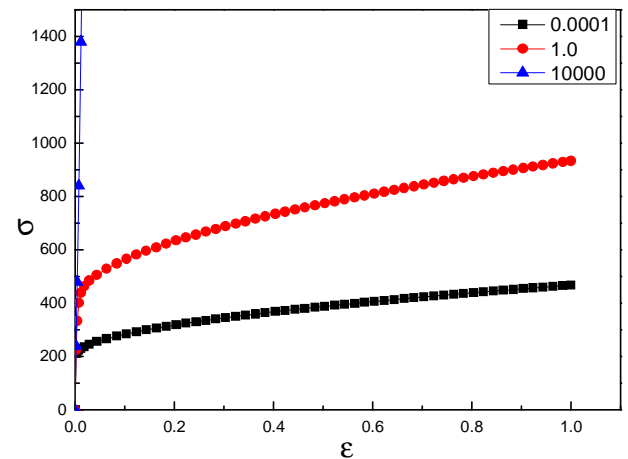

(a) $\epsilon=10^{1}$

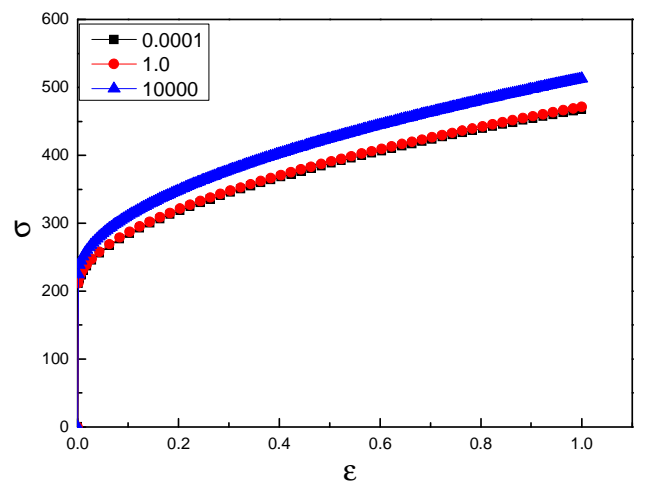

(c) $\epsilon=10^{-2}$

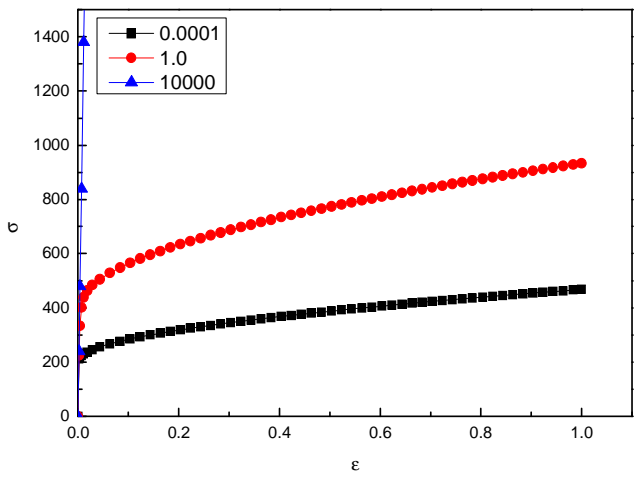

(b) $\epsilon=10^{0}$

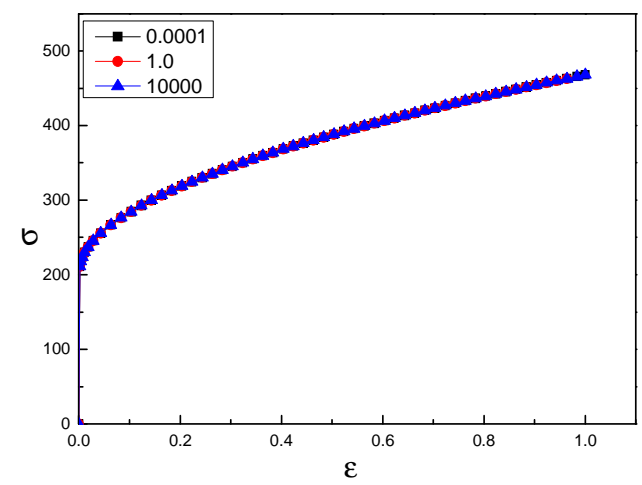

(d) $\epsilon=10^{-6}$

Figure 2. Uniaxial-tensile stress-strain curve

A series of stress-strain curves for each power-law exponent $\epsilon=10^{1}, 10^{0}, 10^{-2}$ and $10^{-6}$ are drawn from the numerical uniaxial tensile test, which are shown, respectively, in Figures 2(a), (b), (c), (d). It can be seen that when the power-law exponent $\epsilon$ is larger than 1.0, the material shows a significant strain rate sensitivity. The mechanical response increases remarkably with the increase of strain rate, when $\epsilon=10^{1}$ and $\epsilon=10^{\circ}$. In addition, when $v^{*}=10^{4}$, the material doesn't yield during the deformation and demonstrates a very high stiffness. When the power-law exponent $\epsilon=10^{-2}$, although the material still shows visco- 
plastic mechanical behavior, the influence of the strain rate on the response decreases. Particularly, when $v^{*}=10^{-4}$ and $v^{*}=10^{0}$, the mechanical response almost coincides. This behavior becomes more obvious when the power-law exponent $\epsilon=10^{-6}$. In this case, all the stress-strain curve coincide and the viscoplastic model is effectively reduced to the elastoplastic Cazacu model.

\section{(b) Uniaxial compressive test}

Owing to the different mechanical behavior of Titanium alloy under compressive stress states and the tensile stress states, a uniaxial compressive benchmark test is used to study the stress integration algorithm of the viscoplastic cazacu model at different strain rates. Similar to the tensile test, a single 8-node brick element (see Figure.3) is loaded with a prescribed displacement. The simulation is also carried out for three different values of $v^{*}$

$$
v^{*}=10^{-4}, 10^{0}, 10^{4}
$$

and four values of $\epsilon=10^{1}, 10^{0}, 10^{-2}, 10^{-6}$ are also studied [11].

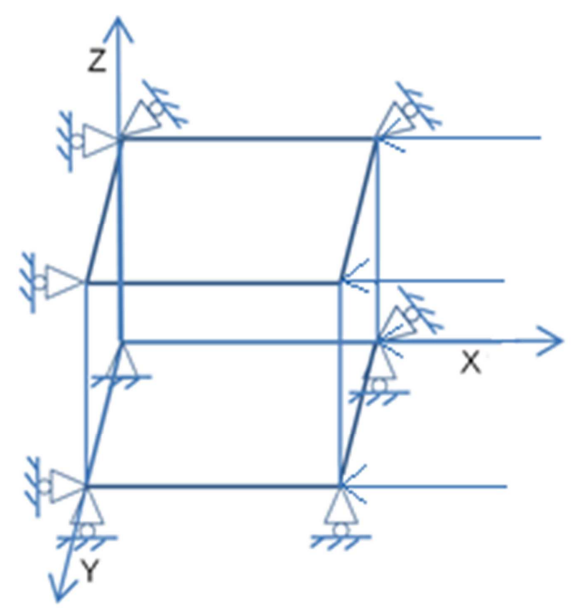

Figure.3 Uniaxial compressive test

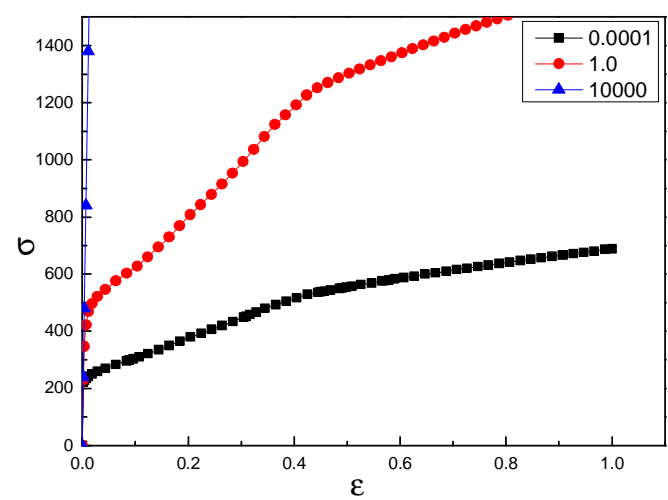

(a) $\epsilon=10^{1}$

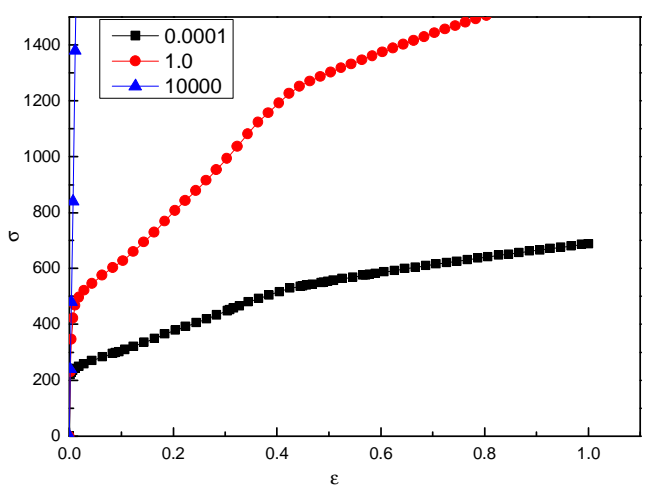

(b) $\epsilon=10^{0}$ 


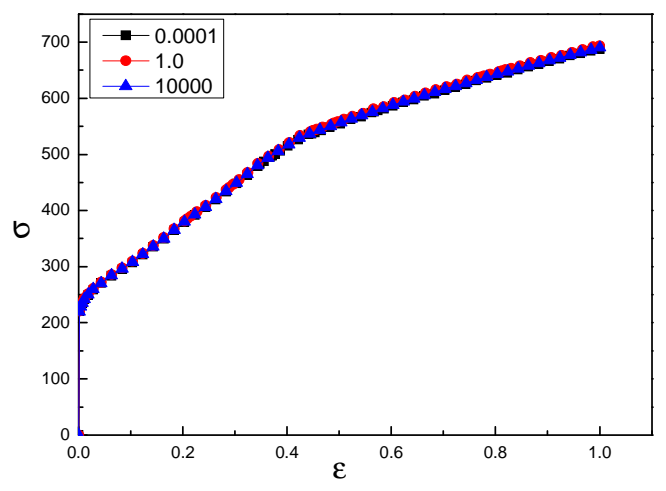

(c) $\epsilon=10^{-2}$

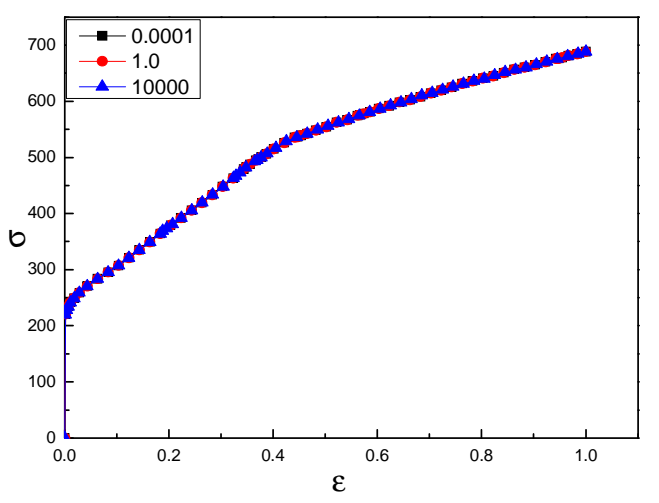

(d) $\epsilon=10^{-6}$

Figure 4. Uniaxial-compressive stress-strain curve

Stress-strain curves for the power-law exponent $\epsilon=10^{1}, 10^{0}, 10^{-2}$ and $10^{-6}$ are also drawn from this numerical uniaxial compressive test, which are shown, respectively, in Figures 4(a), (b), (c), (d). From these, we can see that the mechanical response under compressive stress states also shows strong strain rate sensitivity for high power-law exponent. By comparing with the stress-strain curve under uniaxial tensile stress states, it can be seen that the strain rate sensitivity in compression is higher than the tensile stress state, which can be concluded obtained from the Figure 4 (c). In the compression test, the stress-strain curves coincide for all the strain rates when the power-law exponent $\epsilon=10^{-2}$, however, in the scenario of tensile loading the mechanical response at high strain rate of $10^{4}$ is higher for low $\left(v^{*}=10^{-4}\right)$ and medium $\left(v^{*}=10^{0}\right)$ strain rate.

\subsection{Iso-error maps}

In order to assess the accuracy of the stress integration algorithm, iso-error maps are drawn by standard numerical testing procedure under realistic finite time/strain steps [12]. Using the three-dimensional implementation of the model, we start from a stress point at time $t_{n}, \boldsymbol{\sigma}_{n}$, lying on the yield surface, and a sequence of strain increments is applied corresponding to specified normalised elastic trial stress increments of the form

$$
\Delta \boldsymbol{\sigma}^{\text {trial }}=\frac{\Delta \sigma_{T}}{\sigma_{e q}} \boldsymbol{T}+\frac{\Delta \sigma_{N}}{\sigma_{e q}} \boldsymbol{N}
$$

where $\boldsymbol{N}$ and $\boldsymbol{T}$ are, respectively, the unit (in Euclidean norm) normal and tangent vectors to the yield surface and $\sigma_{e q}$ is the equivalent stress.

Applying increments of trial stress in the tangential and normal direction to the yield surface, the error is evaluated by

$$
\operatorname{ERROR}(\%)=\frac{\sqrt{\left(\sigma_{n}-\sigma_{E X A C T}\right):\left(\sigma_{n}-\sigma_{E X A C T}\right)}}{\sqrt{\sigma_{E X A C T}: \sigma_{E X A C T}}}
$$


where, $\boldsymbol{\sigma}_{n}$ is numerical solution, and $\boldsymbol{\sigma}_{E X A C T}$ is the exact solution. Here, due to the lack of an analytical solution, $\boldsymbol{\sigma}_{E X A C T}$ is assumed to be the stress obtained by sub-incrementation of each stress increment into 1000 steps.

In order to evaluate the accuracy properties and remarkable robustness at different strain rates, several iso-error maps are drawn at low and high strain rates with the non-dimensional factor:

$$
\mu\|\dot{\varepsilon}\|
$$

Set, respectively, to 1 and 1000. In order to investigate the influence of power-law exponent, three values chosen: $10^{0}, 10^{-1}, 0$.

(a) Iso-error maps under a uniaxial tensile stress state

Figures 5 and 6 show the iso-error maps under uniaxial tensile stress state obtained at the aforementioned low and high strain rates for different power-law exponents, $\epsilon$.For the powerlaw exponent $\epsilon=0$, the standard rate-independent Cazacu elasto-plastic model is recovered. The resulting iso-error map in this case is identical to the rate-independent map. For the small power-law exponent, $\epsilon=0.1$, the iso-error map is almost the same as the rate-independent map at the low strain rate (see in Figure 5 (b)). However, the iso-error map changes significantly at the high strain rate (see in Figure 6 (b)). This also occurs for the power-law exponent $\epsilon=10^{\circ}$. It can also be seen that the maximum value of iso-error maps for high power-law exponent is higher than in the rate-independent map; nevertheless, it decreases sharply at high strain rate. The larger the power-law exponent $\epsilon$, the more strain rate sensitivity of the isoerror value. From the maximum iso-error value, it is possible to conclude that the current stress integration algorithm is remarkable robust and can handle effectively any power law exponent $\epsilon$ - from small (corresponding to high rate-sensitivity) to extremely large values (corresponding to effectively rate-independent conditions)_-under low as well as high strain rates.

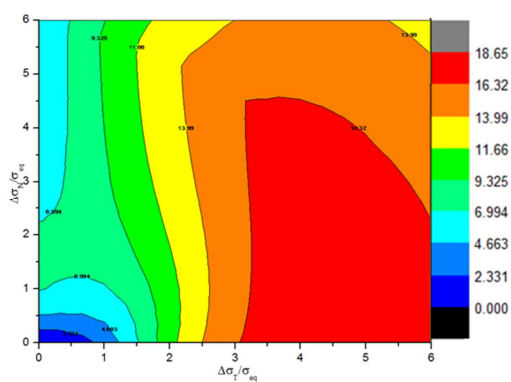

(a)

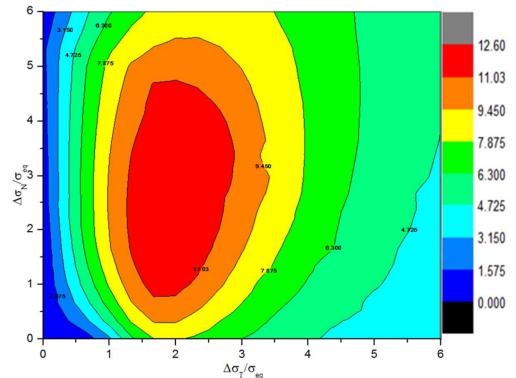

(b)

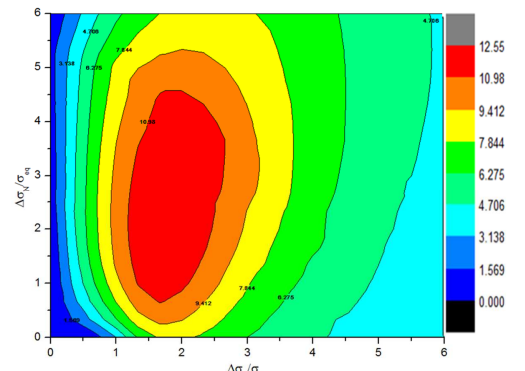

(c)

Figure 5. Iso-error maps under a uniaxial-tensile stress state with $\mu\|\dot{\varepsilon}\|=1.0$. (a) $\epsilon=10^{0}$; (b) $\epsilon=10^{-1}$; (c) $\epsilon=0$ (rate-independent) 


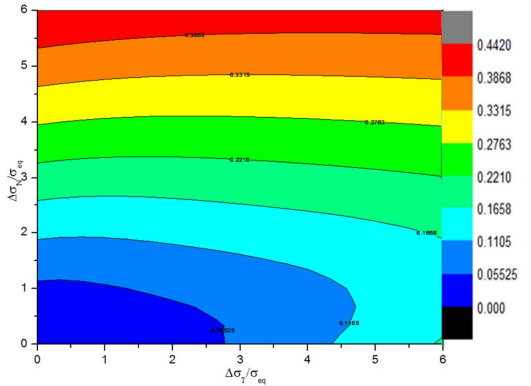

(a)

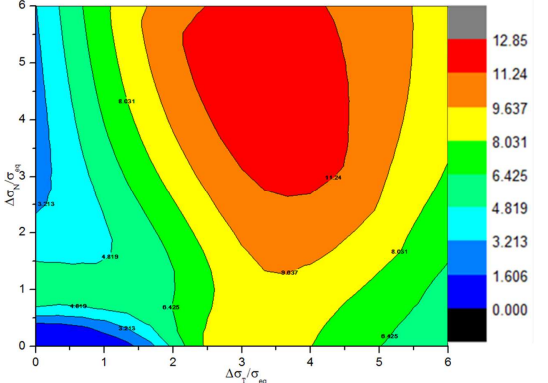

(b)

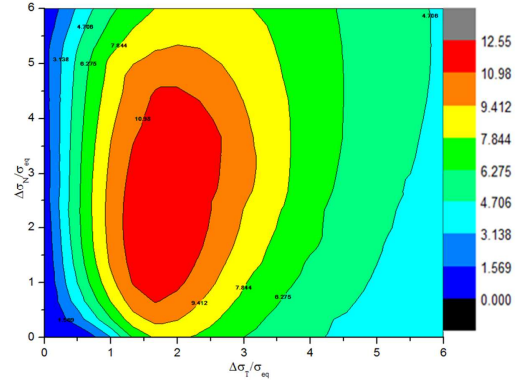

(c)

Figure 6. Iso-error maps under a uniaxial-tensile test with $\mu\|\dot{\varepsilon}\|=1000$. (a) $\epsilon=10^{0}$; (b) $\epsilon=10^{-1}$; (c) $\epsilon=0$ (rate-independent)

(b) Uniaxial-compressive stress state

The iso-error maps under a uniaxial compressive stress state obtained at low and high strain rates for different power-law exponents $(\epsilon)$, which can be seen in Figures 7 and 8 . The same stress integration algorithms and tolerances are applied to draw iso-error maps at uniaxialcompressive stress state. From Figures 7 and 8, the same behavior for the strain rate sensitivity can be observed in the uniaxial compressive stress state. From the comparison between the iso-error maps under uniaxial compressive stress state and under uniaxial tensile stress state, it is clear that the iso-error maps are completely different, which illustrates the importance of the strength differential (SD) effect.

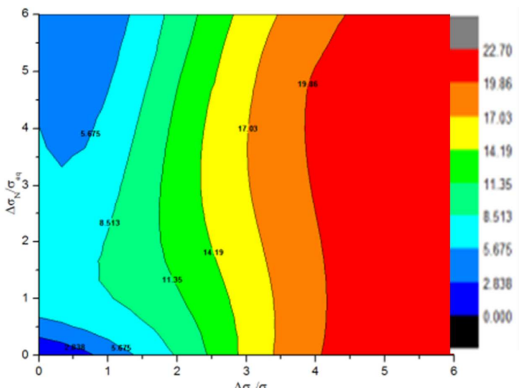

(a)

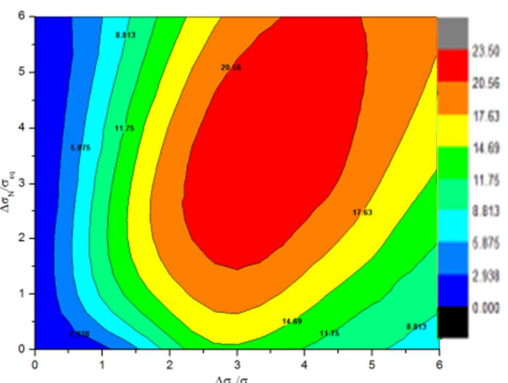

(b)

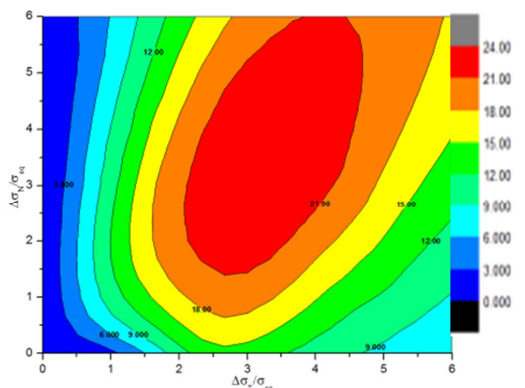

(c)

Figure 7. Iso-error maps at uni-compressive test with $\mu\|\dot{\varepsilon}\|=1$. (a) $\epsilon=10^{0}$; (b) $\epsilon=10^{-1}$; (c) $\epsilon=0$ (rate-independent)

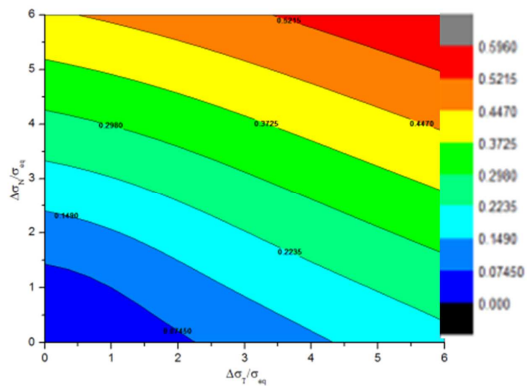

(a)

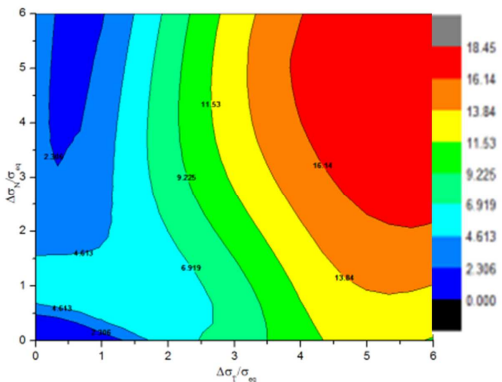

(b)

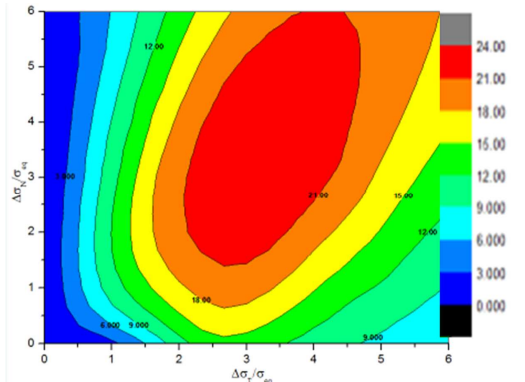

(c)

Figure 8. Iso-error maps at uni-compressive test with $\mu\|\dot{\varepsilon}\|=1000$. (a) $\epsilon=10^{0}$; (b) $\epsilon=$ $10^{-1}$; (c) $\epsilon=0$ (rate-independent) 


\section{CONCLUSION}

In this work, an elastic-viscoplastic constitutive model coupled with Cazacu's yield function has been proposed to simulate the mechanical behavior of metals with an HCP crystal structure at different deformation strain rates. The Closest Point Projection Method (CPPM) is implemented within an implicit quasi-static finite element environment. Two numerical examples show that the integration algorithm can be effectively used at high strain rate even for very high power-exponent. It is also shown that the stress evolution under compressive stress states is higher than under tensile stress states and is more strain rate sensitive.

\section{Acknowledgements}

Financial supported by Portuguese Science and Technology Foundation (FCT), under Grant No. PTDC/EME_TME/105688/2008.

\section{REFERENCES}

[1] Michael E. Nixon, Oana Cazacu, Ricardo A. Lebensohn. Anisotropic response of highpurity a-titanium: Experimental characterization and constitutive modeling. International Journal of Plasticity 26 (2010) 516-532.

[2] A. Rusinek, R. Zaera, J.R. Klepaczko. Constitutive relations in 3-D for a wide range of strain rates and temperatures-application to mild steels. International Journal of Solids and Structures Volume 44, Issue 17, 15 August 2007, Pages 5611-5634.

[3] Oana Cazacu, Frćdćric Barlat. A criterion for description of anisotropy and yield differential effects in pressure insensitive metals. International Jounal of Plasticity 20 (2004) 2027-2045.

[4] Serkan Ertürk. Thermo-mechanical modeling and simulation of magnesium alloys during extrusion process. Gkss Forschungszentrum Geesthacht GMBH. 2009

[5] Michael E. Nixon. Experimental characterization and modeling of the mechanical response of titanium for quasi-static and high strain rate loads. University of Florida, Ph.D thesis. 2008

[6] Lutjering, G., Williams, J. C., 2003. Titanium. Springer, Berlin.

[7] Khan, A. S., A. Pandey, et al. (2011). Mechanical response and texture evolution of AZ31 alloy at large strains for different strain rates and temperatures. International Journal of Plasticity 27(5): 688-706.

[8] Jiang, L., J. J. Jonas, et al. (2007). Twinning and texture development in two Mg alloys subjected to loading along three different strain paths. Acta Materialia 55(11): 3899-3910. 
[9] Plunkett, B., O. Cazacu, et al. (2008). Orthotropic yield criteria for description of the anisotropy in tension and compression of sheet metals. International Journal of Plasticity 24(5): 847-866.

[10] Otto M.Heeres, Akke S.J.Suiker, Rene de Borst. A comparison between the Perzyna viscoplastic model and the consistency viscoplastic model. European Journal of Mechanics 21 (2002) 1-12

[11] de Souza Neto, E. A. (2004). A simple robust numerical integration algorithm for a power-law visco-plastic model under both high and low rate-sensitivity. Communications in Numerical Methods in Engineering 20(1): 1-17.

[12] EA de Souza Neto, D Peric, and DRJ Owen. Computational methods for plasticity theory and applications. John Wiley \& Sons Ltd, 2008. 